\title{
A New Multistate Transition Model For Effect Estimation In Randomized Trials With Treatment Switching And A Cured Subgroup
}

Haixia Hu ( $\sim$ huhx_vero@163.com )

Air Force Medical University

Ling Wang

Air Force Medical University

Chen Li

Air Force Medical University

Wei Ge

Air Force Medical University

Jielai Xia

Air Force Medical University

\section{Research Article}

Keywords: Multistate model, treatment switching, cured subgroup, semi-competing risks, effect estimation

Posted Date: January 3rd, 2022

DOI: https://doi.org/10.21203/rs.3.rs-1183057/v1

License: (c) (1) This work is licensed under a Creative Commons Attribution 4.0 International License. Read Full License 
Title: A New Multistate Transition Model for Effect Estimation in Randomized Trials with Treatment Switching and a Cured Subgroup

Running title: Multistate Transition Model for Treatment Switching

Authors: Haixia $\mathrm{Hu}^{1}$, Ling Wang ${ }^{1}$, Chen $\mathrm{Li}^{1}$, Wei Ge${ }^{1}$, and Jielai Xia ${ }^{1,2}$

1 Department of Health Statistics, Faculty of Preventive Medicine, Air Force Medical University, Xi'an, Shaanxi, China.

2 Correspondence to: Jielai Xia. Department of Health Statistics, Faculty of Preventive Medicine, Air Force Medical University, No.169 Changle West Road, Xi'an 710032, Shaanxi, China. Email: jielaixia@yahoo.com. 


\section{Abstract}

Background: Many methods, including multistate models, have been proposed in the literature to estimate the treatment effect on overall survival in randomized trials with treatment switching permit after the disease progression. Nevertheless, the cured fraction of patients has not been considered. The cured would never experience the progressive disease, but they may suffer death with a hazard comparable to that of people without the disease. With the mix of the cured subgroup, existing methods yield highly biased effect estimation and fail to reflect the truth in uncured patients.

Methods: In this paper, we propose a new multistate transition model to incorporate the cure, progression, treatment switching, and death states during trials. In the proposed model, the probability of cure and the death hazard of the cured are modeled separately. For the not cured patients, the semi-competing risks model is used with the treatment effect evaluated via transitional hazards between states. The particle swarm optimization algorithm is adopted to estimate the model parameters.

Results: Extensive simulation studies have been conducted to evaluate the performance of the proposed multistate model and compare it with existing treatment switching adjustment methods. Results show that in all scenarios, the treatment effect estimation of the proposed model is more accurate than that of existing treatment switching adjustment methods. Besides, the application to diffuse large B-cell lymphoma data has also illustrated the superiority of the proposed model.

Conclusions: The superiority and robustness of the proposed multistate transition model qualify it to estimate the treatment effect in trials with the treatment switching permit after 
progression and a cured subgroup.

24 Keywords: Multistate model; treatment switching; cured subgroup; semi-competing risks;

25 effect estimation 


\section{Background}

In randomized controlled trials (RCT), patients are randomly assigned to either experimental or control groups and followed up until the occurrence of the event of interest or the termination of the trial. In studies comparing the effectiveness of different treatments, progression-free survival (PFS) is often adopted as a surrogate endpoint of overall survival (OS) due to shorter follow-up requirements and well prediction for OS. In some trials with PFS as the primary endpoint, patients in the control group are permitted to switch onto the experimental group after progression if the experimental efficacy is uncovered by existing or external data. The treatment switching allowance is increasingly common, especially in cancer clinical trials [1]. It is driven by ethical and practice concerns [2, 3]. It is ethically recommended to offer patients early access to the new treatment to obtain potential benefits. Besides, the treatment switching permit has the advantage of boosting trial recruitment without impacting on the analysis of short-term endpoint (i.e., PFS), since the balance between groups is not damaged before treatment switching.

However, for studies on the long-term treatment effect (i.e., OS), which is critical for health technology assessment (HTA) decision-making, the treatment switching contributes to considerable confounding [4]. Many statistical methods have been proposed to adjust for treatment switching in the literature. Robins and Tsiatis [5] proposed the rank preserving structural failure time model (RPSFTM) to estimate the treatment effect in trials with treatment switching. Via accelerated failure time (AFT) models, the counterfactual latent failure time of switchers is calculated and the grid search is adopted to obtain the accelerated factor (AF) estimate based on the randomization. In the process of counterfactual failure time calculation, 
White et al. [6] explained the necessity of re-censoring in breaking the dependence of censoring time and treatment. Further, Branson and Whitehead [7] proposed an iterative parameter estimation (IPE) algorithm to adjust for treatment crossover. Similarly, re-censoring is also needed in the IPE algorithm. The difference is that the likelihood-based analysis is used in IPE instead of the rank-test approach in RPSFTM, which greatly accelerates the effect estimation computation. The IPE method has been extended to the prognostic assessment-based treatment switching [8]. Both the RPSFTM and IPE methods are randomization-based and have the key assumption of common treatment effect (CTE). The CTE assumption limits their application. In cases that the CTE assumption is not satisfied, i.e., the treatment effect in switchers is different from the treatment effect in patients initially randomized to the experimental treatment, a two-stage estimation (TSE) method was proposed by Latimer et al [9]. The treatment effect in switchers, which is defined as the switching effect in this paper, is estimated by comparing the survival of switchers and no-switchers in control group after the progressive disease (PD). The time of PD is regarded as the "second baseline". To obtain the unbiased estimate of the switching effect, the "no unmeasured confounders" assumption is required at the second baseline. The inverse probability of censoring weights (IPCW) method [10], an observationalbased method to estimate treatment effect in studies with treatment switching, is also constrained by the "no unmeasured confounders" assumption. Extensive comparisons of these treatment switching adjustment methods have been carried out in the literature [2, 9, 11-15]. A major flaw of these methods is that the treatment effect change with patient status is neglected. Some patients may experience PD before death while some do not. For patients who experienced PD, the treatment effects on OS before and after PD are probably different, which 
has not been accounted for in these methods.

Multistate models have been proposed in the literature to describe the different trajectories to death and the corresponding treatment effect. Specifically, Zeng et al [16] proposed to model the observed PD and death times via a semi-competing risks model. The logistic model was used to model the progression status (i.e., progression or not before death). Semi-parametric hazard models are used in the transitions between randomization to death, randomization to progression, and progression to death. Zhang et al [17] extended Zeng's method with the gamma frailty model to account for dependence between PD and death times and a Bayesian procedure was adopted to estimate the treatment effect. Huang et al [18] adopted copula models to establish the joint distribution of PD and death times subsequently. Chen et al [19] extended Zeng's method to accommodate two-way time-varying switching. With design-based treatment switching in the context of recurrent events data, Chen [20] proposed a semiparametric frailty modeling approach to estimate time-varying effects.

Though gradually refined and improved, the semi-competing risks model has not considered the subgroup for whom the disease is cured. Those patients are insusceptible to PD. It is worth noting that the cured or not is different from the patient disparity in trajectories to death mentioned before. In the semi-competing risks model, some patients may not experience PD due to the censoring of death. However, the cure means that patients would never experience PD even without the censoring of death. With the development of medicine, many diseases, such as early-stage cancers [21], could be clinically curable and patients would never experience PD and would have a life expectancy similar to that of people without the disease. In the analysis of acute graft-versus-host disease (aGVHD), Lee et al [22] took the fraction of 
patients insusceptible to the aGVHD into consideration and proposed a novel multistate model incorporating semi-competing risks model and the cured proportion. Treatment switching has not been considered in Lee's study nevertheless. Zhang et al [23] have also considered the cured fraction to the disease and they adopted a cure rate model for the PD time. But the emphasis of their study was the joint modeling of the longitudinal biomarkers and the survival data, the treatment effect assessment accounting for switching has not been comprehensively considered.

In this paper, we propose a novel multistate transition model which includes the cured fraction of patients, the semi-competing risks between progression and death, and the treatment switching. In the proposed model, logistic models are adopted for the patient heterogeneity (i.e., cured or not) and the treatment switching choice, semi-competing risks model with patientspecific shared frailty is used for the progression and death times, as well as the correlation between them. The treatment effect on cure rate, progression, and death hazard is quantitatively measured using the coefficient of the covariate "group" in each sub-model. The proposed model evaluates the treatment effects on the cured and uncured subgroups separately. The impact of the cured subgroup on the treatment effect estimation in uncured patients is eliminated successfully.

\section{Methods}

\section{Multistate transition model}

In this subsection, we introduce the proposed multistate transition model in detail. Five states are incorporated in the model, including cured, not cured, progression, treatment switching, and death. The transitions between these five states are shown in Figure 1. The cured fraction (bold in Figure 1) is innovatively considered in the proposed model in comparison with 


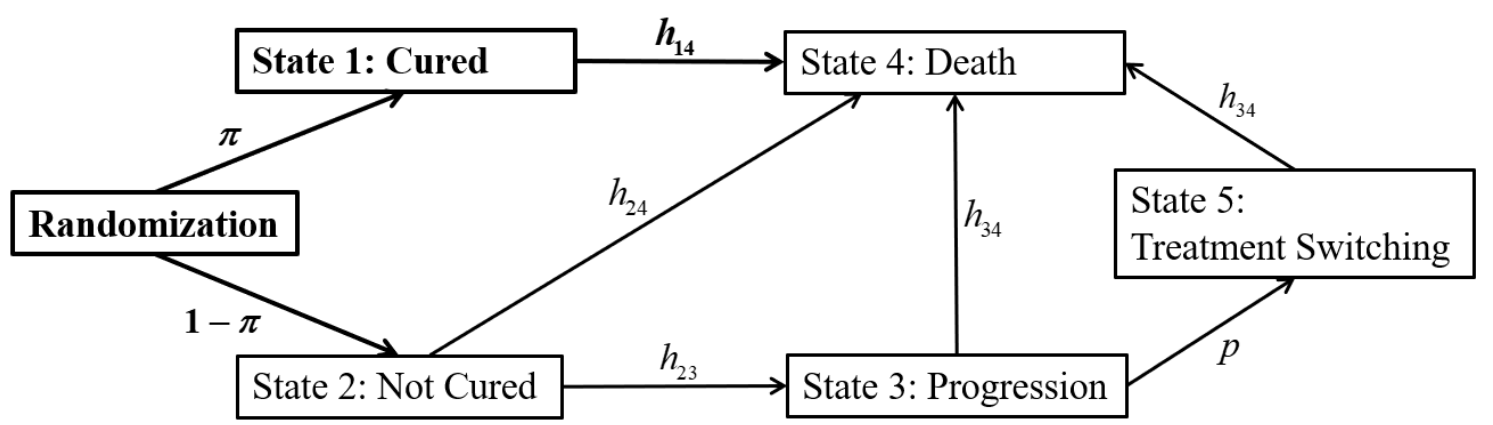

Figure 1. Proposed multistate transition model in which the cured fraction (bold) is innovatively considered.

In RCTs comparing the effectiveness of experimental and control treatments, a proportion of patients might be cured and they would never experience the PD. Let $s$ be the cure indicator with $s=1$ representing the cured patient and $s=0$ representing the not cured patient. The probability of being cured (denoted by $\pi$ ) is expressed as

$$
\pi=P(s=1)=\frac{1}{1+\exp \left[-\left(a_{0}+a_{1} t r t\right)\right]},
$$

where trt is the treatment indicator with $t r t=1$ representing the experimental treatment and $t r t=0$ representing the control treatment. With the specified cure rate of experimental and control treatments (denoted by $\pi_{\exp }$ and $\pi_{c t r}$, respectively), the intercept $\left(a_{0}\right)$ and coefficient $\left(a_{1}\right)$ in equation (1) could be calculated easily. Assume that patients randomized to control treatment are allowed to switch onto the experimental treatment after PD. The treatment switching is voluntary and may be influenced by the time to PD, which is denoted by $t_{P D}$. For example, patients who do well and have longer progression time are advised to stay on the control treatment rather than switching [24]. Similarly, a logistic model is used to establish the probability of treatment switching (denoted by $p$ ), i.e., 


$$
p=P\left(V=1 \mid s=0, \operatorname{trt}=0, \delta_{P D}=1\right)=\frac{1}{1+\exp \left[-\left(b_{0}+b_{1} Q_{t_{P D}}\right)\right]}
$$

where $V$ is the treatment switching indicator with $V=1$ representing switching and $V=0$ otherwise, $\delta_{P D}$ is the indicator for PD occurrence with $\delta_{P D}=1$ representing that the time to PD is observed and $\delta_{P D}=0$ otherwise. $Q_{t_{P D}}$ is an ordinal categorical variable converted by $t_{P D}$, with $Q_{t_{P D}}=1,2,3,4$ representing that the $t_{P D}$ is at $<25 \%, 25 \%-50 \%, 50 \%-75 \%,>70 \%$ quantiles of the progression time, respectively. The ordinal categorical variable $Q_{t_{P D}}$ rather than the original continuous variable $t_{P D}$ is used to describe the impact of the progression time on the treatment switching probability for ease of clinical interpretation. Based on similar studies $[16,22]$ in the literature, we express the transition hazards between states $1-4$ as follows.

$$
\begin{gathered}
h_{14}\left(t_{D} \mid s=1\right)=\gamma \lambda_{14}\left(t_{D}\right), 0<t_{D} \\
h_{23}\left(t_{P D} \mid t r t, s=0\right)=\gamma \lambda_{23}\left(t_{P D}\right) \exp \left(\beta_{23} t r t\right), 0<t_{P D} \\
h_{24}\left(t_{D} \mid t r t, s=0\right)=\gamma \lambda_{24}\left(t_{D}\right) \exp \left(\beta_{24} t r t\right), 0<t_{D} \\
h_{34}\left(t_{P P D} \mid t_{P D}, t r t, s=0\right)=\gamma \lambda_{34}\left(t_{P P D}\right) \exp \left(\beta_{34,1} t r t+\beta_{34,2} V(1-t r t)\right), 0<t_{P D}<t_{D}
\end{gathered}
$$

In equations (3)-(6), $t_{P D}, t_{D}, t_{P P D}$ represent the time to PD, time to death without progression, and time to post-progression death, respectively, $\gamma$ is the patient-specific shared frailty following a gamma distribution with mean one and variance $\theta$, i.e., $G a\left(\theta^{-1}, \theta^{-1}\right)$ [25]. The correlation between the progression time and the death time, as well as the patient heterogeneity, is reflected by the shared frailty. In equation (3), only the baseline hazard is included and the treatment has no effect on the death hazard of the cured subgroup, as we assume that the cured have similar death hazards as people who have never suffered the disease. In equation (6), the coefficient $\beta_{34,1}$ and $\beta_{34,2}$ represent the experimental treatment effect and the treatment switching effect, respectively, on the hazard of post-progression death. Under the assumption 
of CTE, we have $\beta_{34,1}=\beta_{34,2}=\beta_{24}$. For treatments that have a smaller effect on progressed patients, $\beta_{34,1}$ is closer to zero than $\beta_{24}$. In cases that patients switched onto the experimental treatment after progression benefit less compared to patients randomized to the experimental treatment at first, $\beta_{34,2}$ is closer to zero than $\beta_{34,1}$.

\section{Maximum likelihood estimation (MLE) of the treatment effect}

In equations (1)-(6), the cure rate, treatment switching probability, and the transition hazards between states are explicitly modeled. Based on the observed data, the MLE method is used to estimate the parameters in the model.

Suppose a trial with $N$ patients, the observed data for each patient include $\left(t r t_{i}, t_{P D i}, \delta_{P D i}, t_{D i}, \delta_{D i}\right), i=1,2, \ldots, N$, where $\operatorname{trt}_{i}$ is the treatment indicator, $t_{P D i}$ and $t_{D i}$ are the PD and death times, respectively, $\delta_{P D i}$ and $\delta_{D i}$ are the censoring indicator for PD and death, respectively, for the $i$-th patient. The PD time could be censored by both death and trial termination (denoted by $\tau$ ) while the death time could only be censored by $\tau$. For patients who have experienced PD, we have $t_{D i}=t_{P D i}+t_{P P D i}$, where $t_{P P D i}$ is the post-progression death time. Besides, for patients who have experienced PD in the control arm, the treatment switching indicator $V$ illustrates whether he/she switches onto the experimental arm. During the follow-up of the trial, six scenarios may be observed regarding progression, treatment switching and death status (i.e., $\delta_{P D}, V, \delta_{D}$ ), as shown in Table 1 . The likelihood of each status could be calculated accordingly.

Table 1. Observed status indicator and likelihood contribution corresponding to six possible scenarios during the trial. 


\begin{tabular}{llcc}
\hline \multirow{2}{*}{ Status } & \multicolumn{1}{c}{ Description } & $\begin{array}{c}\text { Observed data } \\
\left(\delta_{P D}, V, \delta_{D}\right)\end{array}$ & Likelihood \\
\hline A $\quad$ Observed PD, switching, and death & $(1,1,1)$ & $f_{1}$ \\
B $\quad$ Observed PD, switching, and censored death & $(1,1,0)$ & $f_{2}$ \\
C $\quad$ Observed PD and death without switching & $(1,0,1)$ & $f_{3}$ \\
D $\quad$ Observed PD and censored death without switching & $(1,0,0)$ & $f_{4}$ \\
E $\quad$ Observed death without PD & $(0,-, 1)$ & $f_{5}$ \\
F $\quad$ Censored PD and death & $(0,-, 0)$ & $f_{6}$ \\
\hline
\end{tabular}

According to the trial setting, patients in the control arm are allowed to switch onto the experimental arm only after the occurrence of PD, the switching indicator is not applicable in statuses $\mathrm{E}$ and $\mathrm{F}$ since the PD is not observed. Besides, patients with observed PD are not cured without a doubt, i.e., patients in statuses A-D are not cured $(s=0)$, while patients without observed PD could belong to either the cured or the not cured subgroup. Therefore, the likelihood functions $f_{5} \sim f_{6}$ should be marginalized with respect to the distribution of $s$. Besides, the likelihood functions $f_{1} \sim f_{6}$ are the marginal distributions with respect to the distribution of shared frailty $(\gamma)$, i.e., $f_{k}(t)=\int f_{k}(t \mid \gamma) d \gamma, k=1,2, \ldots, 6$. The corresponding conditional distributions (i.e., $\left.f_{k}(t \mid \gamma), k=1,2, \ldots, 6\right)$ are shown as follows,

$$
f_{1}\left(t_{P D}, t_{P P D} \mid \gamma\right)=P(s=0)\left[S_{24}\left(t_{P D} \mid \gamma\right) S_{23}\left(t_{P D} \mid \gamma\right) h_{23}\left(t_{P D} \mid \gamma\right)\right] P(V=1)^{I(t r t=0)}\left[S_{34}\left(t_{P P D} \mid \gamma\right) h_{34}\left(t_{P P D} \mid \gamma\right)\right]
$$

$$
f_{2}\left(t_{P D}, t_{P P D} \mid \gamma\right)=P(s=0)\left[S_{24}\left(t_{P D} \mid \gamma\right) S_{23}\left(t_{P D} \mid \gamma\right) h_{23}\left(t_{P D} \mid \gamma\right)\right] P(V=1)^{I(t r t=0)} S_{34}\left(t_{P P D} \mid \gamma\right)
$$$$
f_{3}\left(t_{P D}, t_{P P D} \mid \gamma\right)=P(s=0)\left[S_{24}\left(t_{P D} \mid \gamma\right) S_{23}\left(t_{P D} \mid \gamma\right) h_{23}\left(t_{P D} \mid \gamma\right)\right] P(V=0)^{I(t r t=0)}\left[S_{34}\left(t_{P P D} \mid \gamma\right) h_{34}\left(t_{P P D} \mid \gamma\right)\right]
$$$$
f_{4}\left(t_{P D}, t_{P P D} \mid \gamma\right)=P(s=0)\left[S_{24}\left(t_{P D} \mid \gamma\right) S_{23}\left(t_{P D} \mid \gamma\right) h_{23}\left(t_{P D} \mid \gamma\right)\right] P(V=0)^{I(t r t=0)} S_{34}\left(t_{P P D} \mid \gamma\right)
$$$$
f_{5}\left(t_{D} \mid \gamma\right)=P(s=0)\left[S_{23}\left(t_{D} \mid \gamma\right) S_{24}\left(t_{D} \mid \gamma\right) h_{24}\left(t_{D} \mid \gamma\right)\right]+P(s=1)\left[S_{14}\left(t_{D} \mid \gamma\right) h_{14}\left(t_{D} \mid \gamma\right)\right]
$$ 
$f_{6}\left(t_{D} \mid \gamma\right)=P(s=0)\left[S_{23}\left(t_{D} \mid \gamma\right) S_{24}\left(t_{D} \mid \gamma\right)\right]+P(s=1) S_{14}\left(t_{D} \mid \gamma\right)$

where $I(t r t=0)$ is the control treatment indicator, $S_{24}(t \mid \gamma)=\exp \left[-\int_{0}^{t} h_{24}(u \mid \gamma) d u\right]$,

$S_{23}(t \mid \gamma)=\exp \left[-\int_{0}^{t} h_{23}(u \mid \gamma) d u\right] \quad, \quad S_{34}(t \mid \gamma)=\exp \left[-\int_{0}^{t} h_{34}(u \mid \gamma) d u\right] \quad, \quad$ and

$S_{14}(t \mid \gamma)=\exp \left[-\int_{0}^{t} h_{14}(u \mid \gamma) d u\right]$. Assume the transitional baseline hazards are constant, i.e.,

$\lambda_{14}(t)=\lambda_{14}, \lambda_{23}(t)=\lambda_{23}, \lambda_{24}(t)=\lambda_{24}$, and $\lambda_{34}(t)=\lambda_{34}$, with marginal distributions of the

conditional distributions above, the observed data likelihood for

$\Phi=\left(a_{0}, a_{1}, b_{0}, b_{1}, \lambda_{14}, \lambda_{23}, \beta_{23}, \lambda_{24}, \beta_{24}, \lambda_{34}, \beta_{34,1}, \beta_{34,2}, \theta\right)$ is

where $I(t r t=1)$ is the experimental treatment indicator. To maximize $L(\Phi)$ in equation (7),

let

199

$$
\left.L(\Phi)=\prod_{i=1}^{N}\left\{\begin{array}{l}
\left(f_{1}^{\delta_{P D} \delta_{D} V} f_{2}^{\delta_{P D}\left(1-\delta_{D}\right) V} f_{3}^{\delta_{P D} \delta_{D}(1-V)} f_{4}^{\delta_{P D}\left(1-\delta_{D}\right)(1-V)}\right)^{I(t r t=0)} \\
\cdot\left(f_{1}^{\delta_{P D} \delta_{D}} f_{2}^{\delta_{P D}\left(1-\delta_{D}\right)}\right)^{I(t r t=1)}
\end{array}\right] f_{5}^{\left(1-\delta_{P D}\right) \delta_{D}} f_{6}^{\left(1-\delta_{P D}\right)\left(1-\delta_{D}\right)}\right\},
$$

Then, the goal is to find $\Phi$ that maximizes $Z(\Phi)$ in equation (8).

\section{Particle swarm optimization (PSO) algorithm}

method is adopted. PSO algorithm is a population-based stochastic optimization technique

developed by Kennedy and Eberhart [26], inspired by the social behavior of bird flocking or

fish schooling. It has been successfully applied in the area of medicine, such as medical images analysis [27], information clustering [28], disease diagnosis [29], and so on. The flow chart of 


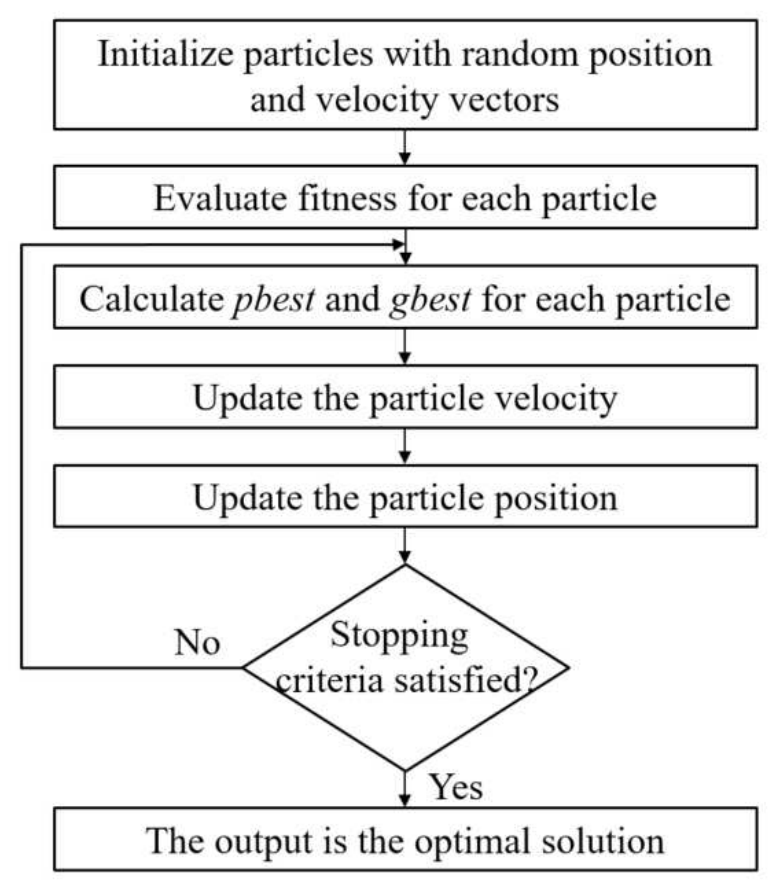

Figure 2. Flow chart of the PSO algorithm [30].

There are five steps in the PSO algorithm.

1) Initialize $M$ solutions, which are named "particles" in PSO, for $\Phi$. There are a position vector and a velocity vector for each particle. The position vector (denoted by $\boldsymbol{\varphi}$ ) represents the candidate solution to maximize $Z(\Phi)$ in equation (8), or similarly, to minimize $-Z(\Phi)$, while the velocity vector (denoted by $\mathbf{v}$ ) represents the direction and amplitude of the position change in the next iteration to search for a better solution. The number of dimensions of the position and velocity vectors is equal to the number of elements in $\Phi$.

2) Calculate the value of the target function to evaluate the fitness of each particle. In the context of this study, calculate $Z\left(\boldsymbol{\varphi}_{m}\right), m=1,2, \ldots, M$ in equation (8).

3) Calculate "pbest" and "gbest", where "pbest" is the best position of each particle in history 
iterations while "gbest" is the best position among all particles in history iterations. The larger the value of $Z(\varphi)$, the better the position of particles.

4) Update the particle velocity and position. For the $m$-th particle, the velocity and position in have observed PD in the control treatment arm, the logistic model is used to fit the treatment 

classified cured patients, observed PD time of classified not cured patients, death time of classified not cured patients without observed PD, and post-progression death time of classified not cured patients with observed PD, respectively. The initial value of $\theta$ is set at 0.5 . Secondly, define the solution search space for each parameter. Specifically, for the parameter $\theta$, the search space is limited within $(0.1,2.5)$, for parameters $\lambda_{14}, \lambda_{23}, \lambda_{24}$ and $\lambda_{34}$, the search space is limited within the scope of crude point estimate multiplied by 0.01 to 100 , while the search space for other parameters is limited within the scope of crude point estimate plus or minus one. Thirdly, generate the random initial position of each particle within the search space of each parameter. The elements in the initial velocity of each particle are generated randomly within $(-0.1,0.1)$.

\section{Results}

\section{Simulation study}

\section{$\underline{\text { Study design }}$} to experimental and control groups and a certain proportion of patients could be cured in both groups. For cured patients, the progression would never happen, the death hazard is small and independent of the treatment. Uncured patients in the control group are permitted to switch onto

261 the experimental group after PD and the switching probability is dependent on the time to PD. parameters are as follows. 


$$
\begin{gathered}
\pi_{\exp }=30 \%, \pi_{c t r}=15 \%, p=30 \%, b_{1}=\log (3), \\
\lambda_{14}=0.0003, \lambda_{23}=0.02, \beta_{23}=\log (0.4), \lambda_{24}=0.005, \beta_{24}=\log (0.4), \\
\lambda_{34}=0.03, \beta_{34,1}=\log (0.4), \beta_{34,2}=\log (0.4), \theta=1 .
\end{gathered}
$$

That is, the cure rates in experimental and control groups are $30 \%$ and $15 \%$, respectively, the values of $a_{0}$ and $a_{1}$ are calculated accordingly. With the switching proportion of $30 \%$ and the value of $b_{1}$, the value of $b_{0}$ could be computed via iterations. Under the setting of $\lambda_{23}, \beta_{23}, \lambda_{24}$ and $\beta_{24}$, about $20 \%$ uncured patients would die without progression. Besides, in this scenario, $\beta_{23}=\beta_{24}=\beta_{34,1}=\beta_{34,2}=\log (0.4)$. That is, the experimental therapy has the same treatment effect on prolonging the PFS and OS, on prolonging OS in patients with or without PD, as well as in switchers.

\section{Control methods for switching adjustment}

To provide context on the performance of the proposed multistate transition model, we present the estimation results of several control methods. The control methods are grouped into simple treatment switching adjustment methods and multistate model methods. For the former, the treatment effect is assumed to be a constant for all patients. Only one parameter is to be estimated for these methods. These methods include:

1) intention to treat (ITT) analysis which ignores the treatment switching;

2) per-protocol analysis which censors the switchers at the PD time (PPcen);

3) per-protocol analysis which excludes the switchers from the analysis dataset (PPexc);

4) RPSFTM method with the treatment effect estimated via grid searching (GE) [5];

5) RPSFTM method with the treatment effect estimated via iteration parameter estimation (IPE) [7].

Re-censoring is considered in GE and IPE methods. Besides, the two-stage estimation (TSE) 
method, which estimates the switching effect and treatment effect separately in two stages [9], is also classified into the category of simple treatment switching adjustment methods in this paper. The multistate model methods highlight the treatment effect heterogeneity across patient subcategories and patient statuses. In this paper, we consider the proposed multistate transition model without considering the shared frailty and Zeng's semi-competing risks model. For comparability, the PSO algorithm is adopted for the parameter estimation of Zeng's method instead of the expectation-maximization (EM) algorithm adopted in their research [16].

\section{$\underline{\text { Investigated scenarios }}$}

To evaluate the robustness of the proposed multistate transition model, as well as the performance sensitivity of control methods concerning different trial settings, the simulation study covers several scenarios. Only one key variable changes across scenarios to show the influence of the variable on the performance of the proposed and control methods. Specifically, based on the basic scenario described in the "Study design" subsection, the changing variables are listed as follows across scenarios.

1) Cure rate: $\pi_{\exp }=\pi_{c t r}=15 \%$ with an equal cure rate between groups; $\pi_{\exp }=30 \%, \pi_{c t r}=15 \%$ with the cure rate ratio being two; and $\pi_{\exp }=45 \%, \pi_{c t r}=15 \%$ with the cure rate ratio being three.

2) Switching proportion: $10 \%$ (low), 30\% (moderate), and 50\% (high).

3) Treatment effect (TE) assumption:

A. CTE satisfied: the treatment effect on OS is the same for switchers and patients initially randomized to experimental treatment, no matter whether the PD happens or not, i.e., $\beta_{24}=\beta_{34,1}=\beta_{34,2}=\log (0.4)$. 
When CTE is not satisfied, two scenarios are considered as follows.

B. The treatment effects on OS are different for patients with or without PD and we assume the protective effect of experimental treatment is larger for patients without $\mathrm{PD}$, i.e.,

4) Sample size: 500, 1000, and 2000.

\section{Performance measures}

The main purpose of this paper is to estimate the experimental treatment effect on OS in

RCTs with treatment switching permission after progression in control group and with a proportion of patients cured in both groups. In multistate models, including the proposed multistate transitional models, the treatment effects on transitions towards different states are described by different parameters $\left(\beta_{23}, \beta_{24}\right.$, and $\left.\beta_{34,1}\right)$. We evaluate the performance of the proposed model and other multistate methods via the accuracy of the estimates of $\beta_{23}, \beta_{24}$, and $\beta_{34,1}$, as well as other parameters. The estimation accuracy is measured by the percentage bias $(\mathrm{PB}(\%))$, mean squared error (MSE), and empirical standard error (SE) of the estimate. Specifically, $\operatorname{PB}(\%)$ is calculated as $(\overline{\hat{\beta}}-\beta) /|\beta| \times 100$, where $\beta$ refers to the true value of parameters and $\overline{\hat{\beta}}$ is the mean of the parameter estimates. The percentage bias is more preferable to the bias because of the different scales of the parameters to estimate. MSE is calculated as $\sum(\hat{\beta}-\beta)^{2} / N_{\text {itr }}$, where $N_{\text {itr }}$ is the number of the simulation replicates. 
TSE) ignore the treatment effect heterogeneity for different patient subcategories or the effect change with the PD status of patients. The treatment effect estimates of these methods reflect the average effect in all patients across all statuses. We evaluate them from two aspects. On the one hand, the treatment effect on death without progression $\left(\beta_{24}\right)$ is served as the true value, since patients who have not progressed are recruited in the trial to evaluate the treatment effect on OS. On the other hand, under each scenario, we simulate data for 1000000 patients without incorporating treatment switching and estimate the average hazard ratio (ave.HR) on OS. The logarithm of the average HR, i.e., $\log ($ ave.HR $)$, is served as the true value to evaluate the performance of the simple treatment switching adjustment methods. For GE, IPE, and TSE methods, the death time data corrected by the estimated AF are used to estimate the HR via a proportional hazard model [6]. It is worth noting that the average treatment effect estimate, i.e., $\log ($ ave.HR $)$, is prone to error because it is obtained via simulation instead of the calculation, but the error is likely to be extremely minimal given the large number of patients simulated [32].

\section{$\underline{\text { Simulation results }}$}

The simulation results are displayed in two parts. In the first part, the multistate models are compared (i.e., proposed multistate transition model with or without shared frailty versus Zeng's semi-competing risks model). In the second part, the performances of simple treatment switching adjustment methods are investigated. In either part, the estimation performances concerning different scenarios are presented. 100 datasets are simulated in each scenario.

Under the basic scenario, the estimation performances of the proposed multistate transition model and Zeng's semi-competing risks model are shown in Table 2. As shown in the third and 
fourth columns, the proposed multistate transition model could provide an almost unbiased estimation of the parameters via the PSO algorithm, especially for the baseline hazards of transitions between states (i.e., $\lambda_{14}, \lambda_{23}, \lambda_{24}$, and $\lambda_{34}$ ). For parameters except $\lambda_{14}$, the estimation bias is less than $4 \%$ of the true value. From the results of the proposed multistate transition model without considering shared frailty (middle part in Table 2), we see that the 356 estimation bias is greatly increased. It is observed that the baseline hazards (i.e., $\lambda_{14}, \lambda_{23}, \lambda_{24}$, and $\lambda_{34}$ ) are underestimated uniformly. The treatment effects are also underestimated from the perspective of HR (i.e., $\exp \left(\beta_{23}\right), \exp \left(\beta_{24}\right)$, and $\exp \left(\beta_{34,1}\right)$ ), while the treatment effect for

359 switchers (i.e., $\left.\exp \left(\beta_{34,2}\right)\right)$ is overestimated. The probabilities of cure and treatment switching are also overestimated. As seen in the last four columns in Table 2, the semi-competing risks model without considering the cured fraction and shared frailty leads to large estimation biases, particularly in the effect estimate on the death without progression (i.e., $\lambda_{24}$ ). The direction of the estimation bias for the semi-competing risks model is the same as that for the multistate transition model without considering shared frailty. Therefore, failing to consider the cured fraction and the shared frailty results in large estimation biases. 
Table 2. Parameter estimation performances of the proposed multistate transition model with or without shared frailty and Zeng's semi-competing risks model under the basic scenario, $N=2000$.

\begin{tabular}{|c|c|c|c|c|c|c|c|c|c|c|c|c|c|}
\hline \multirow{2}{*}{ Parameter } & \multirow{2}{*}{$\begin{array}{l}\text { True } \\
\text { value }\end{array}$} & \multicolumn{4}{|c|}{ Proposed multistate transition model } & \multicolumn{4}{|c|}{ Proposed multistate transition model ${ }^{\gamma-}$} & \multicolumn{4}{|c|}{ Semi-competing risks model } \\
\hline & & Est & PB (\%) & SE & MSE & Est & PB (\%) & $\mathrm{SE}$ & MSE & Est & $\mathrm{PB}(\%)$ & SE & MSE \\
\hline$a_{0}$ & -1.730 & -1.770 & -2.338 & 0.201 & 0.041 & -1.174 & 32.147 & 0.069 & 0.314 & & & & \\
\hline$a_{1}$ & 0.890 & 0.917 & 3.065 & 0.190 & 0.036 & 0.933 & 4.848 & 0.104 & 0.013 & & & & \\
\hline$b_{0}$ & -3.870 & -3.917 & -1.224 & 0.299 & 0.091 & -3.012 & 22.169 & 0.301 & 0.826 & & & & \\
\hline$b_{1}$ & 1.099 & 1.112 & 1.223 & 0.095 & 0.009 & 2.118 & 92.770 & 0.102 & 1.049 & & & & \\
\hline$\lambda_{14}$ & 0.0003 & 0.0003 & -7.285 & $0.001^{\dagger}$ & $0.001^{\dagger}$ & $0.001^{\dagger}$ & -20.342 & $0.001^{\dagger}$ & $0.001^{\dagger}$ & & & & \\
\hline$\lambda_{23}$ & 0.020 & 0.020 & -0.913 & 0.002 & $0.001^{\dagger}$ & 0.013 & -35.298 & 0.001 & $0.001^{\dagger}$ & 0.016 & -21.880 & 0.001 & $0.001^{\dagger}$ \\
\hline$\beta_{23}$ & -0.916 & -0.913 & 0.371 & 0.117 & 0.014 & -0.536 & 41.490 & 0.075 & 0.150 & -0.534 & 41.773 & 0.073 & 0.152 \\
\hline$\lambda_{24}$ & 0.005 & 0.005 & 1.722 & $0.001^{\dagger}$ & $0.001^{\dagger}$ & 0.003 & -36.624 & $0.001^{\dagger}$ & $0.001^{\dagger}$ & 0.002 & -62.394 & $0.001^{\dagger}$ & $0.001^{\dagger}$ \\
\hline$\beta_{24}$ & -0.916 & -0.917 & -0.118 & 0.146 & 0.021 & -0.566 & 38.236 & 0.129 & 0.139 & -0.786 & 14.181 & 0.112 & 0.029 \\
\hline$\lambda_{34}$ & 0.030 & 0.030 & -0.877 & 0.002 & $0.001^{\dagger}$ & 0.019 & -36.511 & 0.001 & $0.001^{\dagger}$ & 0.019 & -36.790 & 0.001 & $0.001^{\dagger}$ \\
\hline$\beta_{34,1}$ & -0.916 & -0.896 & 2.216 & 0.114 & 0.013 & -0.654 & 28.644 & 0.098 & 0.078 & -0.678 & 26.016 & 0.107 & 0.068 \\
\hline$\beta_{34,2}$ & -0.916 & -0.908 & 0.950 & 0.134 & 0.018 & -1.084 & -18.354 & 0.128 & 0.045 & -1.127 & -23.001 & 0.121 & 0.059 \\
\hline$\theta$ & 1.000 & 0.991 & -0.922 & 0.125 & 0.016 & & & & & & & & \\
\hline
\end{tabular}

Est: parameter estimate; $\mathrm{PB}(\%)$ : percentage bias; SE: empirical standard error; MSE: mean squared error. ${ }^{\gamma}$-: multistate transition model without shared frailty. ${ }^{\dagger}$ : values less than 0.001 . 

are shown in Tables S1-S8 of the Appendix 1 in Additional file. On the whole, the performances of the three methods in scenarios of different cure rates, switching proportions, TE assumptions, and sample sizes are similar to that in the basic scenario. The bias of the parameter estimate based on the proposed multistate transition model is consistently small across different scenarios except for the case of a small sample size. When $N=500$ (see Table S8), the estimation bias cannot be ignored. The sample size of 500 might be too small to estimate 13 parameters at once. Besides, for the parameter $\lambda_{14}$, the estimation in some scenarios is not satisfactory due to the impact of extreme estimates. The possible reason is that the true value of $\lambda_{14}$ (i.e., 0.0003 ) is too small to be easily affected by inaccurate estimates. In all scenarios, the parameter transition model. For Zeng's semi-competing risks model, the parameter estimation bias is large death hazard, which amplifies the treatment effect of the experimental therapy. With the change. Multiple factors may contribute to the result and it is hard to figure out. With the 
for all three methods (see Tables S7-S8 and Table 2).

Figure 3 shows $\mathrm{PB}(\%)$ of the parameter estimate with the proposed multistate transition 390 model and simple treatment switching adjustment methods (i.e., ITT, PPcen, PPexc, GE, IPE, 391 and TSE) under scenarios of different cure rates (Figure 3 A1-A2), switching proportions 392 (Figure 3 B1-B2), TE assumptions (Figure 3 C1-C2), and sample sizes (Figure 3 D1-D2). The 393 logarithm of HR for death without progression (i.e., $\beta_{24}$ ) is served as the true value as shown 394 in the left column in Figure 3. Besides, for simple treatment switching adjustment methods, the estimation performance is also evaluated by comparing the estimate with the logarithm of average HR for death across all patients, as shown in the right column in Figure 3. 

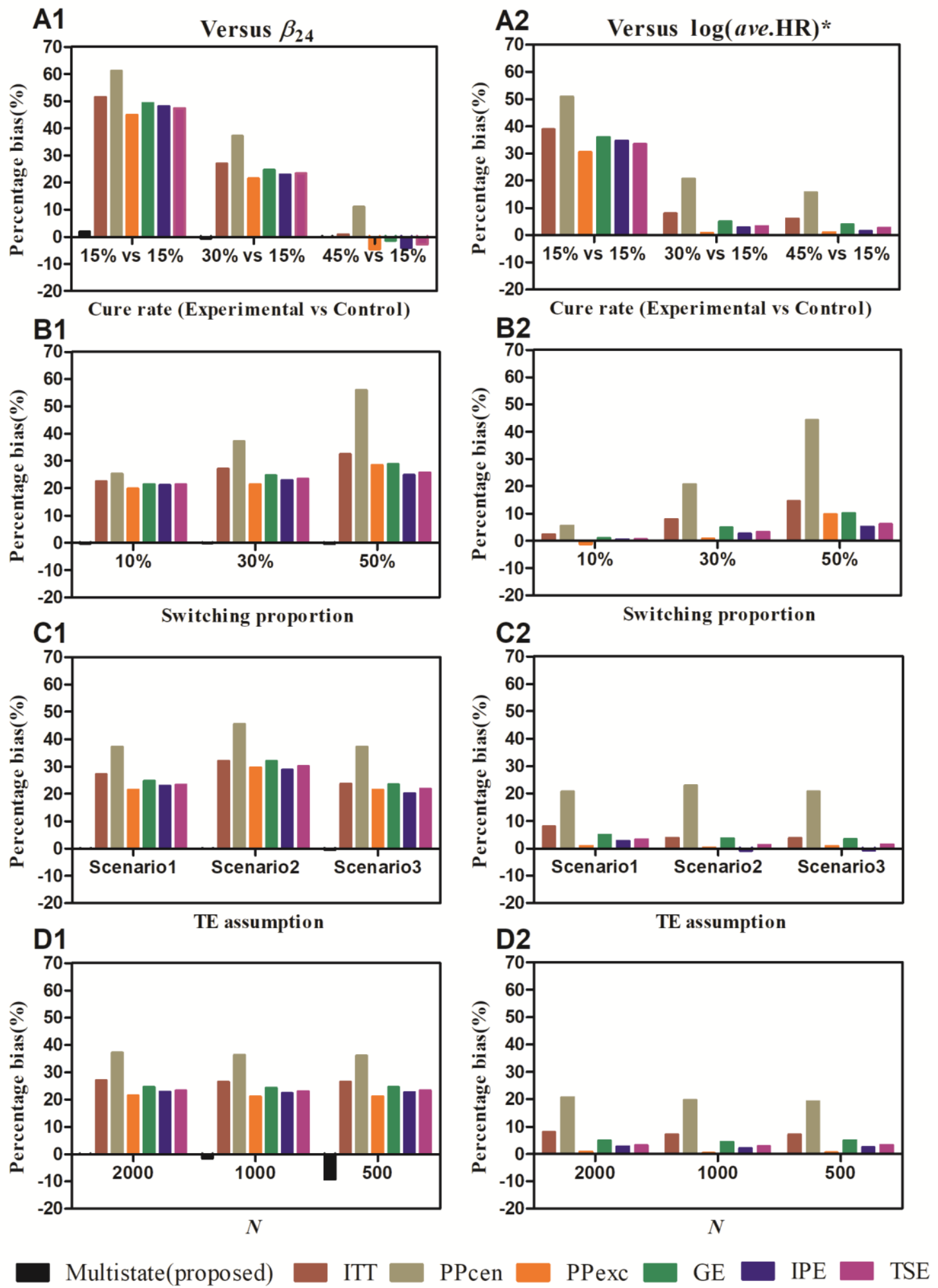

Multistate(proposed) $\square$ ITT $\square$ PPcen

PPexc

TSE

398 Figure 3. $\mathrm{PB}(\%)$ of the estimate using the proposed model and contrast methods under different

399 scenarios.

400 *: Only for control methods (i.e., homogeneity assumption-based methods). TE: treatment effect. In C1-C2, 
scenario 1 is the case that the CTE assumption is satisfied with $\beta_{24}=\beta_{34,1}=\beta_{34,2}=\log (0.4)$; scenario 2 is the case that the CTE assumption is not satisfied with $\beta_{24}=\log (0.4), \beta_{34,1}=\beta_{34,2}=\log (0.6)$; scenario 3 is the case that the CTE assumption is not satisfied with $\beta_{24}=\beta_{34,1}=\log (0.4), \beta_{34,2}=\log (0.6) . N$ : sample size.

As shown in Figure 3, for the estimation of $\beta_{24}$ (left column), the bias of the proposed multistate transition model is much smaller than that of simple treatment switching adjustment methods (i.e., ITT, PPcen, PPexc, GE, IPE, and TSE). Besides, the performance of the proposed multistate transition model is robust with scenarios of different cure rates, switching proportions, and TE assumptions. In cases of small sample sizes, the estimation bias increases but is still acceptable (less than 10\%). For simple treatment switching adjustment methods, the estimation bias of $\beta_{24}$ is much larger. Besides, the bias is positive in most scenarios. That is, the protective treatment effect of the experimental therapy is underestimated. On the one hand, the treatment switching after PD in the control group narrows the death hazard gap between groups. On the other hand, the presence of the cured patients dilutes the treatment effect of the experimental therapy, since the experimental therapy has no effect on the death hazard of cured patients. In comparison with the left column, the estimation biases of simple treatment switching adjustment methods are much smaller in the right column in Figure 3. Because these methods measure the average treatment effect in essence, which is consistent with the true value in the right column. Nevertheless, the performances of ITT and PPcen methods are not satisfactory in the right column. When estimating the average treatment effect with simple treatment switching adjustment methods, the cure rates and switching proportion have large impacts on the estimation performance (see Figure $3 \mathrm{~A} 2$ and B2). When the cure rate in the experimental group increases, the estimation bias for the average treatment effect decreases. Because the average 
death hazard of patients in the experimental group is decreased by the increasing proportion of the cured, the estimated treatment effect is increasing naturally. The estimation bias increases with the switching proportion, especially for ITT, PPcen, and PPexc methods.

The details of the estimation bias variations with the cure rate, switching proportion, TE assumption, and sample size for simple treatment switching adjustment methods and proposed multistate transition model are as follows.

The simulations in Figure $3 \mathrm{~A} 1$ are carried out under the scenario of $N=2000$, CTE assumption satisfied, and switching proportion of $30 \%$. It is observed that the estimation bias of the proposed multistate transition model is consistently small (less than $2 \%$ ) in different cure rate scenarios. For simple treatment switching adjustment methods, the estimation bias of $\beta_{24}$ changes greatly with cure rates. In the scenario of $15 \%$ vs $15 \%$ (i.e., the cure rates are equal in the experimental and control groups), the estimation biases of $\beta_{24}$ based on simple treatment switching adjustment methods are extremely large. Because the death hazard of cured patients is independent of the received therapy, the equal proportions of the cured in experimental and control groups dilutes the treatment effect of the experimental therapy. The treatment effect is greatly underestimated. In scenarios of $30 \%$ vs $15 \%$ and $45 \%$ vs $15 \%$, the cure rate in the experimental group increases, the treatment effect underestimation is gradually eased and the estimation bias of $\beta_{24}$ is decreased. Under the scenario of $45 \%$ vs $15 \%$, influenced by the large cure rate in the experimental group, the treatment effect is overestimated for PPexc, GE, IPE, and TSE methods.

As shown in Figure 3 B1, with the increase of the switching proportion, the estimation bias of all methods increases. The bias of the proposed multistate transition model is much 
smaller than that of the simple treatment switching adjustment methods. With a sample size of 2000 , the bias of the former is less than $1 \%$ while the biases of the latter methods are larger than 20\%. The estimation biases of ITT and PPcen methods are more sensitive to the switching proportion.

In Figure $3 \mathrm{C}$, the $\mathrm{CTE}$ assumption is satisfied in scenario 1 (i.e., the treatment effect on OS is a constant before and after progression across all uncured patients) while the CTE assumption is not satisfied in the other two scenarios. It is observed that the proposed multistate transition model performs well in all scenarios. The estimation bias of simple treatment switching adjustment methods increases in scenario 2. Because in scenario 2, the treatment effect in progressed patients and switchers is smaller, the average treatment effect decreases. Then the treatment effect underestimation is exacerbated. In comparison with scenario 1 , the treatment effect in switchers is smaller in scenario 3, i.e., the switchers benefit less in comparison with the patients who have been randomized to the experimental group from the beginning. The estimation biases of ITT, GE, IPE, and TSE methods decrease in scenario 3 . Because the decreased switching effect attenuates the impact of treatment switching on OS. Besides, we find that the estimation biases of PPcen and PPexc methods are unchanged in scenario 3 compared to that in scenario 1 . That is because the observation after treatment switching is excluded in analysis, the switching effect does not impact the estimation of these two methods.

Figure 3 D1 shows the estimation bias versus the sample size. It is observed that the proposed multistate transition model is more sensitive to the sample size. Because there are much more parameters to estimate in the multistate transition model. The estimation bias 
increases with the decrease of sample size. By contrast, the performance of simple treatment switching adjustment methods is less affected by the sample size. Nevertheless, the estimation bias of the proposed multistate transition model is smaller than that of simple treatment switching adjustment methods in cases of small sample sizes.

\section{Case study}

Diffuse large B-cell lymphoma (DLBCL) is a curable lymphoma [33]. The standard treatment for DLBCL consists of cyclophosphamide, doxorubicin, vincristine, and prednisone (CHOP) chemotherapy with anti-CD20 antibody rituximab (R) [34]. It has been proved that about $45 \%$ of DLBCL patients would be cured with R-CHOP treatments [35]. However, patients with treatment failure after R-CHOP often have a poor outcome [36]. Therefore, it is reasonable to allow patients who progressed with R-CHOP treatment to switch onto the experimental arm in RCT testing the effectiveness of a new treatment for DLBCL.

Assume a new treatment for DLBCL with the cure rate of $50 \%$ and the HR of 0.6 in comparison with R-CHOP treatment, we generated simulation datasets of the RCT comparing the new treatment and R-CHOP. Patients who are progressed in R-CHOP arm are allowed to switch onto the new treatment arm in the simulated RCT. The parameter setting of the simulation data is based on Coiffer's research [35]. More details are elaborated in Appendix 2 of the Additional file. With the switching proportion of $10 \%, 30 \%$, and $50 \%$, the sample size of 2000,1000 , and 500, the bias of the HR estimates of the new treatment obtained by the proposed multistate transitional model, Zeng's semi-competing risks model, ITT, PPcen, PPexc, GE, IPE, and TSE methods are shown in Figure 4. It shows that based on one dataset, the estimation bias of the proposed model is much smaller in comparison with the other methods. With the increase 
of the switching proportion, the estimation bias of contrast methods increases, while the estimation bias of the proposed model is robust. Under the scenario of $N=1000$, the estimation bias of the proposed model, as well as that of contrast methods, is larger, it could be attributed to the sample error of the simulated dataset.
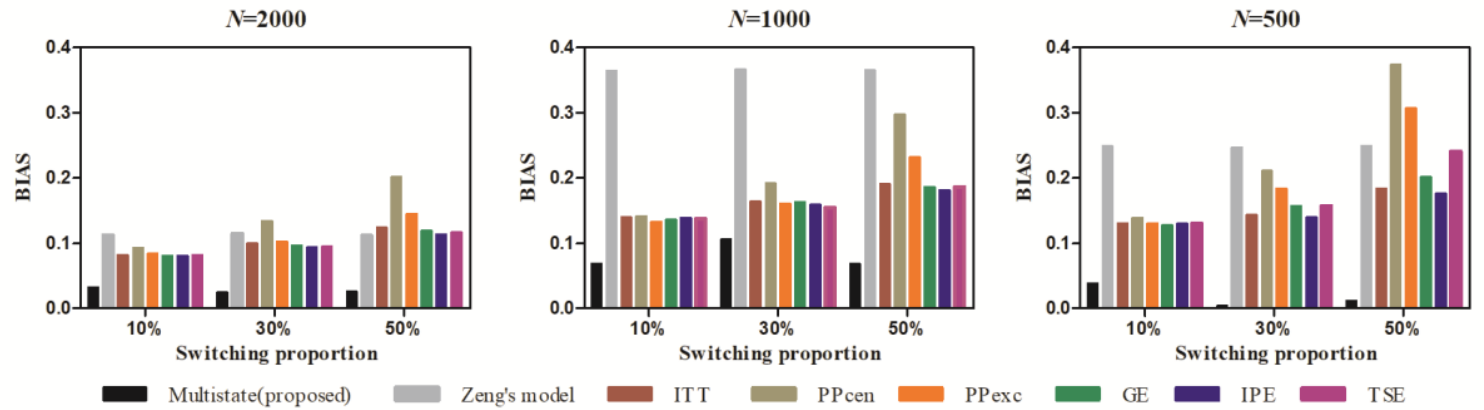

Figure 4. HR estimation bias of the proposed model and contrast methods based on the DLBCL dataset.

\section{Discussion}

To estimate the treatment effect of experimental therapy in RCTs with treatment switching permit in the presence of a cured subgroup, we propose a new multistate transition model in which the disease cure, progression, treatment switching, and death are accommodated. The proposed model hopes to separately quantify the treatment effect on the cure rate, progression hazard, and death hazard with or without progression. There are three trajectories to death in the model, including death for the cured to whom the progression would never happen, death without progression for the uncured, and death after progression for the uncured. The experimental effects on different trajectories are estimated separately in the model. Simulation studies under various scenarios show the good estimation performance and robustness of the proposed model. The results of comparisons with other methods illustrate the superiority of the 
proposed model.

As a multistate model, Zeng's semi-competing risks model is included in the simulation as a control. The main difference between the proposed method and Zeng's method is that the cured fraction and patient-specific frailty are not considered in Zeng's method. Simulation results show that failing to incorporate the cured fraction in the model leads to large estimation biases. The effect estimation based on the proposed model without considering shared frailty is also biased. Therefore, when there is a cured subgroup, the proposed model is preferable. The shared frailty connects the progression and death times and describes the patient heterogeneity at the same time, which makes the model more scientific, rigorous, and rational.

Different from the proposed multistate transition model, some existing methods, including ITT, PPcen, PPexc, GE, IPE, and TSE methods, ignore the multiple trajectories to death in patients. These methods assume that the treatment effect keeps the same in all patients, and the possible change of the treatment effect after progression is also neglected. We call them simple treatment switching adjustment methods in this paper. As shown in the simulation study, the treatment effect estimates of these methods are more close to the effect averaged over all patients and all disease stages. When aiming to estimate the treatment effect on the hazard of one specific transition, for example, the death hazard in patients without progression, simple treatment switching adjustment methods bring about large biases. The bias varies with the cure rate, switching proportion, treatment effect, and switching effect. Therefore, these methods are incapable of providing specific treatment effect estimations on transition hazards between states. Instead, they provide average treatment effect estimation, which could not meet the needs of clinical practice in some cases. Besides, among these simple treatment switching adjustment 
methods, the PPcen method produces the largest bias when estimating the average treatment effect. The GE, IPE, and TSE methods perform better in adjusting the treatment switching and estimating the treatment effect on OS. Many studies have been conducted in the literature to compare these methods, details are not elaborated here since it is not the core of this paper. Readers interested are referred to $[2,9,11-14]$.

The multistate transition model proposed in this paper accommodates the possible cured fraction of patients innovatively, which makes the model more applicable. The shared frailty modeled by gamma distribution accounts for the individual heterogeneity of patients and dependence of progression and death times, which improves the generality of the proposed model. The limitation of the study is that the possible dropout or loss to follow-up and covariates that might influence the progression and death hazard have not been considered in the model, the extension on these aspects is under consideration.

\section{Conclusions}

The superiority and robustness of the proposed multistate transition model qualify it to estimate the treatment effect in trials with the treatment switching permit after progression and a cured subgroup.

\section{List of abbreviations}

AF: accelerated factor; AFT: accelerated failure time; aGVHD: acute graft-versus-host disease; CTE: common treatment effect; DLBCL: diffuse large B-cell lymphoma; EM: expectationmaximization; GE: grid estimation; HR: hazard ratio; HTA: health technology assessment; IPCW: inverse probability censoring weights; IPE: iterative parameter estimation; ITT: intention-to-treat; MSE: mean squared error; OS: overall survival; PB: percentage bias; PD: 
progressive disease; PFS: progression-free survival; PP: per-protocol; PSO: particle swarm optimization; R-CHOP: rituximab, cyclophosphamide, doxorubicin, vincristine, and prednisone; RCT: randomized controlled trial; RPSFTM: rank preserving structural failure time model; SE: standard error; TE: treatment effect.

\section{Declarations}

Ethics approval and consent to participate: Not applicable.

Consent for publication: Not applicable.

Availability of Data and Materials: Parameter settings for the simulated DLBCL dataset used in the case study are publicly available in Additional file.

Competing interests: The authors declare that they have no competing interests.

Funding: This work was supported by the National Nature Science Foundation of China (grant no. $81773553,81973141,81803328)$.

Authors' contributions: HH and JX proposed the conception and designed the work. HH and LW performed the data analysis and drafted the manuscript. CL and WG participated in the results interpretation and manuscript revision. All the authors reviewed and approved the final manuscript.

\section{Additional files}

Additional file.pdf contains the simulation results of the proposed multistate transitional model and Zeng's semi-competing risks model under other scenarios (Appendix 1) and the parameter setting consideration for the simulated DLBCL dataset used in the case study (Appendix 2).

\section{Acknowledgement}

The authors would like to thank Dr. Xueqian Wang in Tsinghua University for his helpful 
suggestions which significantly improved the quality of the paper.

574

575

576

577

578

579

580

581

582

583

584

585

586

587

588

589

590

591

592

593

594

\section{References}

1. Yeh J, Gupta S, Patel SJ, Kota V and Guddati AK. Trends in the crossover of patients in phase III oncology clinical trials in the USA. eCancerMedicalScience. 2020;14:1142.

2. Latimer NR, Abrams KR, Lambert PC, Crowther MJ, Wailoo AJ, Morden JP, et al. Adjusting survival time estimates to sccount for treatment switching in randomized controlled trials - an economic evaluation context. Med Decis Making. 2014;34:387-402.

3. Motzer RJ, Escudier B, Oudard S, Hutson TE, Porta C, Bracarda S, et al. Efficacy of everolimus in advanced renal cell carcinoma: a double-blind, randomised, placebocontrolled phase III trial. the Lancet. 2008;372:449-56.

4. Latimer NR. Treatment switching in oncology trials and the acceptability of adjustment methods. Expert Rev Pharm Out. 2015;15:561-4.

5. Robins JM and Tsiatis AA. Correcting for non-compliance in randomized trials using rank preserving structural failure time models. Commun Stat-Thero M. 1991;20:2609-31.

6. White IR, Babiker AG, Walker S and Darbyshire JH. Randomization-based methods for correcting for treatment changes: examples from the Concorde trial. Stat Med. $1999 ; 18: 2617-34$.

7. Branson M and Whitehead J. Estimating a treatment effect in survival studies in which patients switch treatment. Stat Med. 2002;21:2449-63.

8. Shao J, Chang $\mathrm{M}$ and Chow S. Statistical inference for cancer trials with treatment switching. Stat Med. 2005;24:1783-90.

9. Latimer NR, Abrams KR, Lambert PC, Crowther MJ, Wailoo AJ, Morden JP, et al. 
Adjusting for treatment switching in randomised controlled trials - A simulation study and a simplified two-stage method. Stat Methods Med Res. 2014;26:724-51.

10. Robins JM and Finkelstein DM. Correcting for noncompliance and dependent censoring in an aids clinical trial with inverse probability of censoring weighted (IPCW) log-rank tests. Biometrics. 2000;56:779-88.

11. Morden JP, Lambert PC, Latimer N, Abrams KR and Wailoo AJ. Assessing methods for dealing with treatment switching in randomised controlled trials: a simulation study. BMC Med Res Methodol. 2011;11:4.

12. White I. Methods for handling treatment switching: rank-preserving structural nested failure time models, inverse-probability-of-censoring weighting, and marginal structural models. Report, MRC Biostatistics Unit, UK, February 2012.

13. Watkins C, Huang X, Latimer N, Tang Y and Wright EJ. Adjusting overall survival for treatment switches: commonly used methods and practical application. Pharm Stat. $2013 ; 12: 348-57$

14. Latimer NR, Abrams KR, Lambert PC, Morden JP and Crowther MJ. Assessing methods for dealing with treatment switching in clinical trials: A follow-up simulation study. Stat Methods Med Res. 2016;27:765-84.

15. Montané L, Ferranti D, Chabaud S, Pujade-Lauraine E, Pérol D and Paoletti X. Assessment of post-switch endpoints in randomized clinical trials. Revue d'Épidémiologie et de Santé Publique. 2018;66:S130.

16. Zeng D, Chen Q, Chen MH and Ibrahim JG. Estimating treatment effects with treatment switching via semicompeting risks models: an application to a colorectal cancer study. 
Biometrika. 2012;99:167-84.

17. Zhang Y, Chen M, Ibrahim JG, Zeng D, Chen Q, Pan Z, et al. Bayesian gamma frailty models for survival data with semi-competing risks and treatment switching. Lifetime Data Anal. 2014;20:76-105.

18. Huang CH, Chen YH, Wang JL and Wang M. Semiparametric copula-based analysis for treatment effects in the presence of treatment switching. Stat Med. 2020;39:2936-48.

19. Chen Q, Zhang F, Chen M and Cong XJ. Estimation of treatment effects and model diagnostics with two-way time-varying treatment switching: an application to a head and neck study. Lifetime Data Anal. 2020;26:685-707.

20. Chen Q, Zeng D, Ibrahim JG, Akacha M and Schmidli H. Estimating time-varying effects for overdispersed recurrent events data with treatment switching. Biometrika. 2013;100:339-54.

21. Jia X, Sima CS, Brennan MF and Panageas KS. Cure models for the analysis of time-toevent data in cancer studies. J Surg Oncol. 2013;108:342-7.

22. Lee C, Lee SJ and Haneuse S. Time-to-event analysis when the event is defined on a finite time interval. Stat Methods Med Res. 2020;29:1573-91.

23. Zhang F, Chen M, Cong XJ and Chen Q. Assessing importance of biomarkers: A Bayesian joint modelling approach of longitudinal and survival data with semi-competing risks. Stat Model. 2021;21:30-55.

24. Henshall C, Latimer NR, Sansom L and Ward RL. Treatment switching in cancer trials: issues and proposals. Int J Technol Assess. 2016;32:167-74.

25. Xu J, Kalbfleisch JD and Tai B. Statistical analysis of illness-death processes and 
semicompeting risks data. Biometrics. 2010;66:716-25.

26. Kennedy J and Eberhart R. Particle Swarm Optimization. Proceedings of ICNN'95international conference on neural networks. IEEE. 1995;4:1942-8.

27. Abdel-Basset M, Fakhry AE, El-henawy I, Qiu T and Sangaiah AK. Feature and Intensity Based Medical Image Registration Using Particle Swarm Optimization. J Med Syst. 2017;41:197.

28. Patibandla RSML, Rao BT, Krishna PS and Maddumala VR. Medical data clustering using particle swarm optimization method. Journal of Critical Reviews. 2020;7:363-7.

29. Beheshti Z, Shamsuddin SMH, Beheshti E and Yuhaniz SS. Enhancement of artificial neural network learning using centripetal accelerated particle swarm optimization for medical diseases diagnosis. Soft Comput. 2014;18:2253-70.

30. Amrane Y and Kouba NEY. A Multiobjective optimal VAR dispatch using FACTS devices considering voltage stability and contingency analysis. In: Deo R, Samui P and Roy SS, editors. Predictive Modelling for Energy Management and Power Systems Engineering. Amsterdam: Elsevier; 2021. pp. 1-26.

31. Bansal J, Singh P, Saraswat M, Verma A, Jadon SS and Abraham A. Inertia Weight Strategies in Particle Swarm Optimization. In: Third World Congress on Nature and Biologically Inspired Computing, Salamanca, Spain, 19-21 Oct 2011, pp. 633-640. New York: IEEE.

32. Latimer NR, White IR, Abrams KR and Siebert U. Causal inference for long-term survival in randomised trials with treatment switching: Should re-censoring be applied when estimating counterfactual survival times? Stat Methods Med Res. 2018;28:2475-93. 
661

662

663

664

665

666

667

668

669

670

671

672

673

674

675

676

677

33. Friedberg JW and Fisher RI. Diffuse Large B-Cell Lymphoma. Hematol Oncol Clin N. 2008;22:941-52.

34. Goda JS, Lewis SC, Laskar S, Kannan S, Khanna N, Jain H, et al. A phase III randomized controlled trial of radiation dose optimization in non-Hodgkin lymphoma-diffuse large Bcell lymphoma (DOBL study): Study protocol and design. Cancer reports. 2019;2:e1161.

35. Coiffier B, Lepage E, Briere J, Herbrecht R, Tilly H, Bouabdallah R, et al. CHOP chemotherapy plus rituximab compared with CHOP alone in elderly patients with diffuse large-B-cell lymphoma. N Engl J Med. 2002;346:235-42.

36. Sehn LH, Salles G. Diffuse Large B-Cell Lymphoma. N Engl J Med. 2021;384:842-58.

\section{Figure Legends}

Figure 1. Proposed multistate transition model in which the cured fraction (bold) is innovatively considered.

Figure 2. Flow chart of the PSO algorithm.

Figure 3. $\mathrm{PB}(\%)$ of the estimate using the proposed model and contrast methods under different scenarios.

Figure 4. HR estimation bias of the proposed model and contrast methods based on the DLBCL dataset. 


\section{Supplementary Files}

This is a list of supplementary files associated with this preprint. Click to download.

- Additionalfile.pdf 